\title{
Exploring the factors of workplace injury and chronic illness in return-to-work outcomes
}

\author{
Hafez Hussain \\ Social Security Organisation, Malaysia. hafez.hussain@perkeso.gov.my
}

Objective: The study aims to explore factors of workplace injury and chronic illnesses patients in a return to work program which are associated with return to work outcomes. Design: A retrospective cohort study. Setting: Return to Work Department, Social Security Organisation, Malaysia.

Method: All participants of the Return to Work Rehabilitation Program who were absent from work due to workplace injury and chronic illnesses between January 2008 and December 2013 with no other history of injury were included. The main outcome of the study was the number in days from day one of injury prior to return of gainful employment. Hierarchical multiple regression methods were used to determine the identified factors that influence the return to work outcome.

Results: An initial study with data from January 2008 and December 2011 of cases with occupational injury showed $66 \%(\mathrm{n}=1,552)$ of the participants had returned to work. The average number of days to return to work was 201 days. Hierarchical multiple regression analysis identified duration of referral to the Return to Work rehabilitation program, monthly salary, Whole Person Impairment ratings, DASS 21 anxiety and DASS 21 stress scale as factors that influenced return to work, explaining $32 \%$ of the variance. However an analysis of the remaining cases such as cases with chronic illnesses will be included in the final results of this study.

Conclusion: Currently, a total of $66 \%$ participants had returned to employment after occupational injury. It is essential to understand the complex pathway from the point of injury and illnesses until returning to work in order to develop more effective return to work practices. 